\title{
Article
}

\section{Time-Resolved XUV Absorption Spectroscopy and Magnetic Circular Dichroism at the Ni $M_{2,3}$-Edges}

\author{
Marcel Hennes ${ }^{1, *}\left(\mathbb{C}\right.$, Benedikt Rösner $^{2} \oplus$, Valentin Chardonnet ${ }^{1}$, Gheorghe S. Chiuzbaian ${ }^{1} \oplus$, Renaud Delaunay ${ }^{1}$, \\ Florian Döring ${ }^{2}$, Vitaliy A. Guzenko ${ }^{2}{ }^{(0)}$, Michel Hehn ${ }^{3}$, Romain Jarrier ${ }^{1}$, Armin Kleibert ${ }^{2}{ }^{(}$, Maxime Lebugle ${ }^{2}$, \\ Jan Lüning ${ }^{1}$, Gregory Malinowski ${ }^{3}$, Aladine Merhe ${ }^{1}$, Denys Naumenko ${ }^{4}$, Ivaylo P. Nikolov ${ }^{4}$, \\ Ignacio Lopez-Quintas ${ }^{4}\left(\mathbb{D}\right.$, Emanuele Pedersoli ${ }^{4}\left(\mathbb{D}\right.$, Tatiana Savchenko ${ }^{2}$, Benjamin Watts $\left.{ }^{2}{ }^{(}\right)$, Marco Zangrando ${ }^{4}(\mathbb{D}$, \\ Christian David ${ }^{2}$, Flavio Capotondi ${ }^{4}$, Boris Vodungbo ${ }^{1}$ and Emmanuelle Jal ${ }^{1, *} \mathbb{C}$
}

1 Sorbonne Université, CNRS, Laboratoire de Chimie Physique-Matière et Rayonnement, LCPMR, 75005 Paris, France; valentin.chardonnet@gmail.com (V.C.); gheorghe.chiuzbaian@upmc.fr (G.S.C.); renaud.delaunay@upmc.fr (R.D.); romain.jarrier@upmc.fr (R.J.); jan.luning@helmholtz-berlin.de (J.L.); Alaaeldinemerhe@gmail.com (A.M.); boris.vodungbo@upmc.fr (B.V.)

2 Paul Scherrer Institut, PSI 5232 Villigen, Switzerland; Benedikt.Roesner@psi.ch (B.R.); florian.doering@psi.ch (F.D.); vitaliy.guzenko@psi.ch (V.A.G.); armin.kleibert@psi.ch (A.K.); maxime.lebugle@gmail.com (M.L.); Tatiana.Savchenko@psi.ch (T.S.); benjamin.watts@psi.ch (B.W.); christian.david@psi.ch (C.D.)

3 Institut Jean Lamour, Université de Lorraine, 54011 Nancy, France; michel.hehn@univ-lorraine.fr (M.H.); gregory.malinowski@univ-lorraine.fr (G.M.)

4 Elettra-Sincrotrone Trieste SCpA, 34149 Basovizza, Trieste, Italy; denys.naumenko@tugraz.at (D.N.); ivaylo.nikolov@elettra.eu (I.P.N.); ilopezquintas@usal.es (I.L.-Q.); emanuele.pedersoli@elettra.eu (E.P.); marco.zangrando@elettra.eu (M.Z.); flavio.capotondi@elettra.eu (F.C.)

* Correspondence: marcel.hennes@sorbonne-universite.fr (M.H.); emmanuelle.jal@sorbonne-universite.fr (E.J.)

check for updates

Citation: Marcel, H.; Benedikt, R.; Valentin, C.; Gheorghe, S.C.; Renaud, D.; Florian, D.; Vitaliy, A.G.; Michel,

H.; Romain, J.; Armin, K.; et al.

Time-Resolved XUV Absorption Spectroscopy and Magnetic Circular Dichroism at the Ni $\mathrm{M}_{2,3}$-edges. Appl. Sci. 2021, 11, 325. https://doi.org/ 10.3390/app11010325

Received: 29 November 2020 Accepted: 21 December 2020 Published: 31 December 2020

Publisher's Note: MDPI stays neutral with regard to jurisdictional clai$\mathrm{ms}$ in published maps and institutional affiliations.

Copyright: $\odot 2020$ by the authors. Licensee MDPI, Basel, Switzerland. This article is an open access article distributed under the terms and conditions of the Creative Commons Attribution (CC BY) license (https:// creativecommons.org/licenses/by/ $4.0 /)$.

\begin{abstract}
Ultrashort optical pulses can trigger a variety of non-equilibrium processes in magnetic thin films affecting electrons and spins on femtosecond timescales. In order to probe the charge and magnetic degrees of freedom simultaneously, we developed an X-ray streaking technique that has the advantage of providing a jitter-free picture of absorption cross-section changes. In this paper, we present an experiment based on this approach, which we performed using five photon probing energies at the $\mathrm{Ni} M_{2,3}$-edges. This allowed us to retrieve the absorption and magnetic circular dichroism time traces, yielding detailed information on transient modifications of electron and spin populations close to the Fermi level. Our findings suggest that the observed absorption and magnetic circular dichroism dynamics both depend on the extreme ultraviolet (XUV) probing wavelength, and can be described, at least qualitatively, by assuming ultrafast energy shifts of the electronic and magnetic elemental absorption resonances, as reported in recent work. However, our analysis also hints at more complex changes, highlighting the need for further experimental and theoretical studies in order to gain a thorough understanding of the interplay of electronic and spin degrees of freedom in optically excited magnetic thin films.
\end{abstract}

Keywords: ultrafast spectroscopy; femtomagnetism; XUV-FEL; magnetic circular dichroism

\section{Introduction}

Since its discovery by Beaurepaire and coworkers in 1996 [1], the phenomenon of laser-induced ultrafast demagnetization has attracted worldwide attention and initiated an entirely new research field [2]. However, despite more than 20 years of ongoing experimental and theoretical work, the mechanisms underlying the femtosecond magnetization dynamics in ferromagnetic films following ultrafast optical excitation remain intensively debated [3-7]. The problem is interesting from a fundamental perspective-to date, it is not clear how angular momentum is transferred between the spin and electron systems and the crystalline lattice on sub-picosecond time scales-but it is also technologically 
relevant, as using light to steer magnetization on sub-ps timescales might pave the way for ultrafast all-optical spintronics and data storage [8-10]. Recent developments illustrating the application potential of ultrafast optical excitations include, for example, the creation of artificial neural networks [11], or the demonstration that focusing grating couplers can be used for photonic-electronic integration with magnetoresistive random access memory technology [12].

X-ray experiments are an ideal tool to study the dynamics of the spin, electron, and phonon sub-systems, since they combine high spatial resolution and element selectivity. In general, time-resolved scattering and absorption experiments mainly focus on spin and lattice dynamics, yielding information about superdiffusive spin currents [13-16], the role of spin-orbit coupling [17], the interaction of different magnetic sublattices [18], and the link between lattice and spin dynamics [19-21]. So far, only few studies have simultaneously looked at electron and spin dynamics [17,22-24]. Indeed, performing pure absorption measurements at X-ray free electron lasers (FELs) has been challenging, due to intrinsic fluctuations of the photon parameters and the lack of a reliable and linear incident intensity monitor [25]. However, motivated by time-resolved absorption experiments performed at femtoslicing facilities $[17,22]$, as well as time- and spin-resolved photoelectron spectroscopy at high harmonic generation (HHG) sources [26,27], new technical advances were recently made to probe X-ray absorption dynamics using FEL [25,28-31] and HHG [32,33], thereby laying the ground for a systematic analysis of charge and spin dynamics [30,34].

Within this context, the investigation of the impact of the probing energy on the measured absorption and magnetic circular dichroism (MCD) dynamics has attracted increasing interest [35-37]. In a recent study, Yao et al. [34] provided experimental and theoretical evidence for an electronic and magnetic elemental absorption resonance redshift on ultrafast timescales. Unfortunately, the use of an HHG setup in this study [34] results in photon energies separated by approximately $3 \mathrm{eV}$, which impedes a detailed investigation of the absorption edge itself. To circumvent this shortcoming, we employed X-ray streaking at the extreme ultraviolet (XUV)-FEL at FERMI in Italy, to probe five energies at the $\mathrm{Ni}$ $M_{2,3}$-edges (spanning from 64 to $68 \mathrm{eV}$ ). Our results confirm that absorption and MCD spectra are shifting but also hint at a more complicated scenario involving changes in spectra shapes after optical excitation. This calls for additional systematic experimental studies and in-depth theoretical calculations.

\section{Experiment}

The sample used in our experiment is a $\mathrm{Ni}(20 \mathrm{~nm}) / \mathrm{Fe}_{50} \mathrm{Ni}_{50}(10 \mathrm{~nm}) / \mathrm{Ni}(20 \mathrm{~nm})$ trilayer deposited via magnetron sputtering on top of $\mathrm{Ta}(3 \mathrm{~nm})$-covered $\mathrm{Si}_{3} \mathrm{~N}_{4}(30 \mathrm{~nm})$ membranes. A final $\mathrm{Pt}(3 \mathrm{~nm})$ capping was used to prevent oxidation (Figure 1). The magnetic layers are polycrystalline and exhibit an in-plane anisotropy with a square magnetic hysteresis loop and a coercive field of $8 \mathrm{mT}$. Note that the presence of the $\mathrm{Fe}_{50} \mathrm{Ni}_{50}$ layer was required for a different femtomagnetism study but is not relevant for the outcome of the present work. Indeed, we would like to emphasize that $\mathrm{Ni}$ atoms in the alloy layer make up a total equivalent thickness of only $5 \mathrm{~nm}$, to be compared with $40 \mathrm{~nm}$ of pure Ni. Alloyed Ni thus only contributes $\simeq 10 \%$ to the total absorption signal. For comparison, we used NiFe layers with equal thickness placed at different depths (e.g., $\mathrm{Ni}(10 \mathrm{~nm}) / \mathrm{NiFe}(10 \mathrm{~nm}) / \mathrm{Ni}(30 \mathrm{~nm})$ ). Measurements on these samples yielded the same absorption time traces.

The experiments, based on our recently developed X-ray streaking technique [29], were performed at the DiProI end-station [38] of the seeded XUV-FEL at FERMI in Elettra, Trieste [39]. The setup employed in our study is shown in Figure 1. As described in detail previously [29], our approach is based on the use of a nearly collimated XUV beam with circular polarization, which illuminates an off-axis zone-plate. The converging first order emanating from the zone-plate passes through the sample and its intensity distribution is collected by a charge-coupled device (CCD) camera (sample camera). As shown in Figure 1, the photons detected at the bottom of this camera (yellow line) have traveled a longer path than the ones arriving at the top (green line). This results in a 2.6 ps time difference at 
the zone plate focus, which becomes spatially encoded in the sample camera. Note that optical geometry-induced contributions to the temporal resolution are as low as $2.5 \mathrm{fs}$ and can be neglected here. To measure the shot-to-shot variations of the incident $X$-ray pulses, a second CCD detector (reference camera) is used to record the diverging first negative order. By dividing the sample camera with the reference camera picture, we obtain a measure for the transmission of the sample $T=I / I_{\text {in }}$, where $I$ is the photon intensity after, and $I_{\text {in }}$ the one before the sample. Angular integration of the intensity ratio pictures [29] eventually yields the transmission time traces shown in Figure 2a.

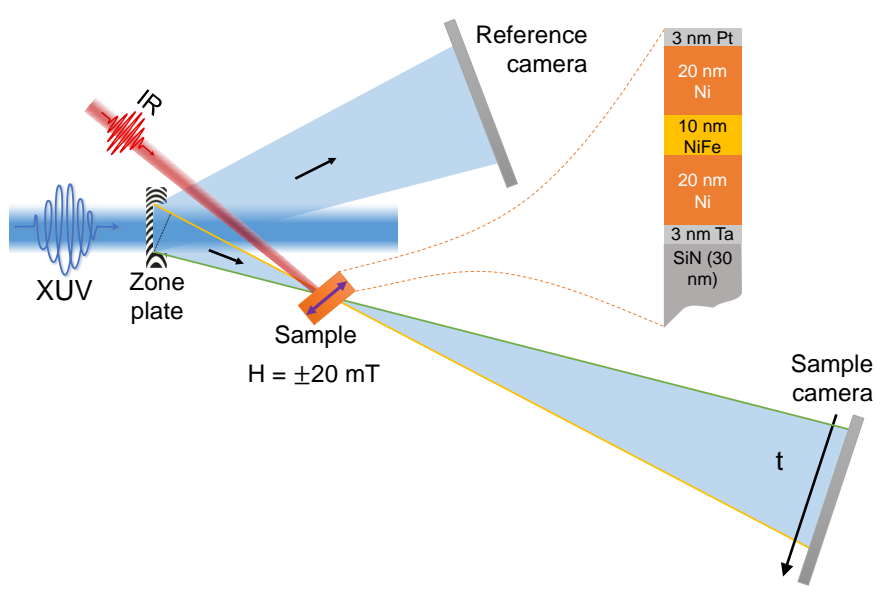

Figure 1. Experimental setup and sample composition. A circularly polarized extreme ultraviolet (XUV) beam is diffracted using a zone plate. The positive, first diffraction order is focused on an in-plane magnetized sample, which is pumped using linear infrared pulses. The sample camera records the intensity transmitted through the sample $I$, resulting in a spatial encoding of the time traces. The reference camera records the incident intensity $I_{\text {in }}$.

In order to be sensitive to the in-plane magnetization of the Ni layer, the sample was tilted by $18^{\circ}$ with respect to the incident beam. To ensure saturation of the magnetization for each pump-probe measurement, an in-plane magnetic field of $\pm 20 \mathrm{mT}$ was applied by using an electromagnet. The sample was excited by an $800 \mathrm{~nm}$ infrared (IR) laser delivering $60 \mathrm{fs}$ pulses using an incident fluence of $5 \mathrm{~mJ} / \mathrm{cm}^{2}$, and probed by circularly polarized $70 \mathrm{fs}$ XUV pulses, with an intensity at the sample plane well below the excitation regime [40]. In the sample plane, the size of the IR spot was $\simeq 250 \times 165 \mu \mathrm{m}^{2}$ (FWHM), significantly larger than the XUV spot size $\simeq 130 \times 70 \mu \mathrm{m}^{2}$ (FWHM). The experiment was performed at $50 \mathrm{~Hz}$. To probe the absorption and MCD around the Ni $M_{2,3}$ resonances, we used five probing XUV wavelengths with energies of $64.6,65.7,66.3,66.7$ and $68 \mathrm{eV}$. For each energy, we recorded CCD images by accumulation of 150 to 250 shots with and without an IR pump for both applied magnetic field directions and both XUV helicities. Note that the CCDs employed in our study cannot be read out at $50 \mathrm{~Hz}$, and that we use the same number of accumulated shots on the reference and the sample camera to perform the normalization. For symmetry reasons, reversing the XUV probe beam helicity is equivalent to a reversal of the applied magnetic field direction. This allows us to average the measured curves, resulting in two transmitted intensities $T^{ \pm}=\frac{I^{ \pm}}{I_{\mathrm{in}}}$. We grouped the data such as to obtain a data point every $30 \mathrm{fs}$, and the error bars presented in all our figures reflect this grouping and averaging procedure.

Typical transmitted intensity time traces are plotted in Figure 2a for an XUV energy of $66.7 \mathrm{eV}$ and both external field directions $\mathrm{H}^{+}$(dark green) and $\mathrm{H}^{-}$(light green). As expected, the thin film is excited on sub-ps timescales; the difference between $T^{+}$and $T^{-}$ is quickly decreasing after $t=0$, which reflects the strong transient modifications of the magnetic properties of the system. 

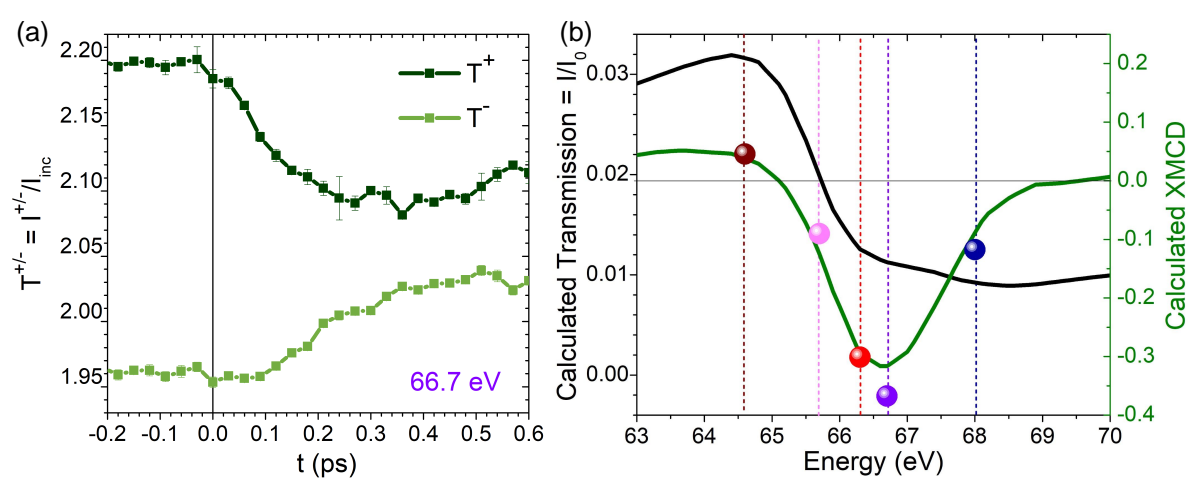

Figure 2. Characteristic pump-induced changes of $T^{+}$and $T^{-}$on fs timescales and static energydependent transmission and magnetic circular dichroism (MCD). (a) Transmitted intensity time traces measured for both applied field directions obtained for $E=66.7 \mathrm{eV}$. (b) Calculated transmission (black) and MCD (green) compared with experimental static MCD (colored dots).

To quantify these changes, $T^{ \pm}$data can be used to calculate the absorption $\mu$ and the $M C D$, which are related to the electronic occupation and to the spin state of our magnetic layer, respectively. We therefore use $\mu^{ \pm} \propto-\ln T^{ \pm}, \mu=\frac{\mu^{+}+\mu^{-}}{2} \propto \frac{\ln \left(T^{+} \cdot T^{-}\right)}{2}$ and $M C D=\mu^{+}-\mu^{-} \propto \ln \frac{T^{-}}{T^{+}}$[41]. Quantities extracted from pumped data will be denoted $T^{ \pm}(t), \mu^{ \pm}(t), \mu(t)$ and $M C D(t)$, while static data will be labeled $T_{0}^{ \pm}, \mu_{0}^{ \pm}, \mu_{0}$ and $M C D_{0}$. In order to compare data measured using different XUV energies, we need to look at the relative changes $\frac{\Delta \mu^{ \pm}(t)}{\mu_{0}^{ \pm}}, \frac{\Delta \mu(t)}{\mu_{0}}$ and $\frac{\Delta M C D(t)}{M C D_{0}}$. Here, the prefix $\Delta$ is used as a short notation for a subtraction of the static signal, e.g., $\Delta \mu(t)=\mu(t)-\mu_{0}$.

Due to different IR filters used in front of the cameras, experimental transmission data are only known up to a prefactor. This can be seen in Figure 2a, where the transmission is superior to 1 , which is of course at odds with the fact that the beam intensity is weakened via absorption. To correct this artifact, we use the calculated transmission $T_{\text {th }}$ shown in Figure $2 \mathrm{~b}$ (dark curve) to rescale our data and obtain the proper relative absorption. The calculated transmission and MCD curves presented in Figure $2 b$ have been obtained using tabulated values (CXRO website [42]) for $\mathrm{Si}_{3} \mathrm{~N}_{4}, \mathrm{Ta}$, and $\mathrm{Pt}$, which were combined with literature data for $\mathrm{Ni}$ and $\mathrm{Fe}$ [43]. Comparison of the calculated MCD signal (green curve in Figure $2 \mathrm{~b}$ ) with our static experimental $M C D_{0}$ (colored dots) yields good agreement for each energy scrutinized in the present study (Figure 2b) (note that the experimental MCD spectrum does not need to be rescaled since the constant pre-factors cancel out as shown in Equation (1)). The following equations summarize our approach, and all data presented in the remainder of this work are based on these calculations:

$$
\begin{aligned}
\frac{\Delta \mu^{ \pm}(t)}{\mu_{0}^{ \pm}} & =\frac{\ln \left(\frac{T_{0}^{ \pm}}{T^{ \pm}(t)}\right)}{\ln \left(\alpha \cdot T_{0}^{ \pm}\right)} \\
\frac{\Delta \mu(t)}{\mu_{0}} & =\frac{\ln \left(\frac{T_{0}^{+} \cdot T_{0}^{-}}{T^{+}(t) \cdot T^{-}(t)}\right)}{\ln \left(\alpha^{2} \cdot T_{0}^{+} \cdot T_{0}^{-}\right)} \quad \text { with } \alpha=\frac{2 T_{\text {th }}}{T_{0}^{+}+T_{0}^{-}} \\
\frac{\Delta M C D(t)}{M C D_{0}} & =\frac{\ln \left(\frac{T_{0}^{+} \cdot T^{-}(t)}{T^{+}(t) \cdot T_{0}^{-}}\right)}{\ln \left(\frac{T_{0}^{-}}{T_{0}^{+}}\right)}
\end{aligned}
$$




\section{Results}

Figure 3 shows the relative absorption for both field directions, $\frac{\Delta \mu^{ \pm}(t)}{\mu_{0}^{ \pm}}$obtained using 5 different XUV wavelengths. For all these probing energies, we observe an increase of the relative absorption for majority electrons $\left(H^{+}\right.$, dark green), while the relative minority electron absorption $\left(H^{-}\right.$, light green) increases for $64.6,65.7$ and $66.3 \mathrm{eV}$ and decreases for 66.7 and $68 \mathrm{eV}$. Thus, the evolution of the minority electron $\left(H^{-}\right.$, light green) absorption with time (and, to a lesser extent, the majority electron adsorption) depends on the probing energy. Note that these changes result from a modification of available states before and after optical excitation. For probing energies below the Fermi level (66.2 eV for Ni [44]) the absorption increase corresponds for example to a depletion of electrons (i.e., more free states available), similarly, for probing energies above the Fermi level (namely $66.7 \mathrm{eV}$ and $68 \mathrm{eV}$ ), a decrease of absorption indicates that the initial free states are now occupied by excited electrons. In addition, Figure 3 shows that transient absorption changes for different applied fields do not systematically start at $t=0$ : the same dynamics are observed for both field directions during the first tens of fs after optical excitation for $E=64.6,65.7$, and $66.3 \mathrm{eV}$ (highlighted grey regions). At first glance, this is in line with the recent observation of a delay between ultrafast electronic and magnetic dynamics [30,34]. For $E=65.7 \mathrm{eV}$, a closer look at the first $100 \mathrm{fs}$ reveals a crossing between $\mathrm{H}^{+}$and $\mathrm{H}^{-}$curves. As we will explain in the discussion section, we believe that this is the signature of a MCD spectrum shift.

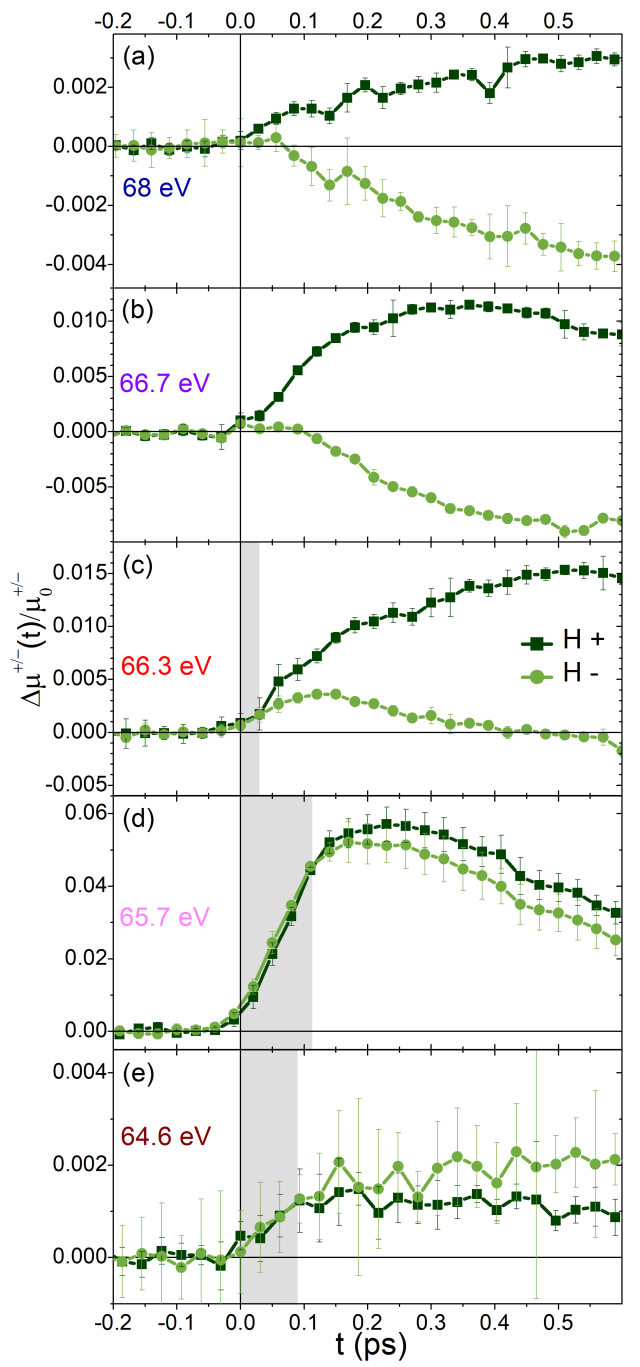

Figure 3. Relative absorption time traces for both field directions: $\mathrm{H}^{+}$shown in dark green and $\mathrm{H}^{-}$ shown in light green using XUV probing energies equal to (a) $68 \mathrm{eV}$ (b) $66.7 \mathrm{eV}$ (c) $66.3 \mathrm{eV}$ (d) $65.7 \mathrm{eV}$ and (e) $64.6 \mathrm{eV}$. 
In order to compare the results obtained for different probing energies and to further quantify the electronic and magnetic dynamics, we have plotted the relative absorption and the relative MCD in Figure 4a,b, respectively. Figure 4a shows $\frac{\Delta \mu(t)}{\mu_{0}}$ (vertical axis) as a function of the delay (left axis) and probing energies (right axis). As a reminder, the static absorption curve is shown in the background (dotted black line) with the corresponding probing energies (dotted colored line). Figure $4 \mathrm{~b}$ shows $\frac{\triangle M C D(t)}{M C D_{0}}$ which is directly proportional to the relative magnetization changes as a function of the time delay for all XUV energies.
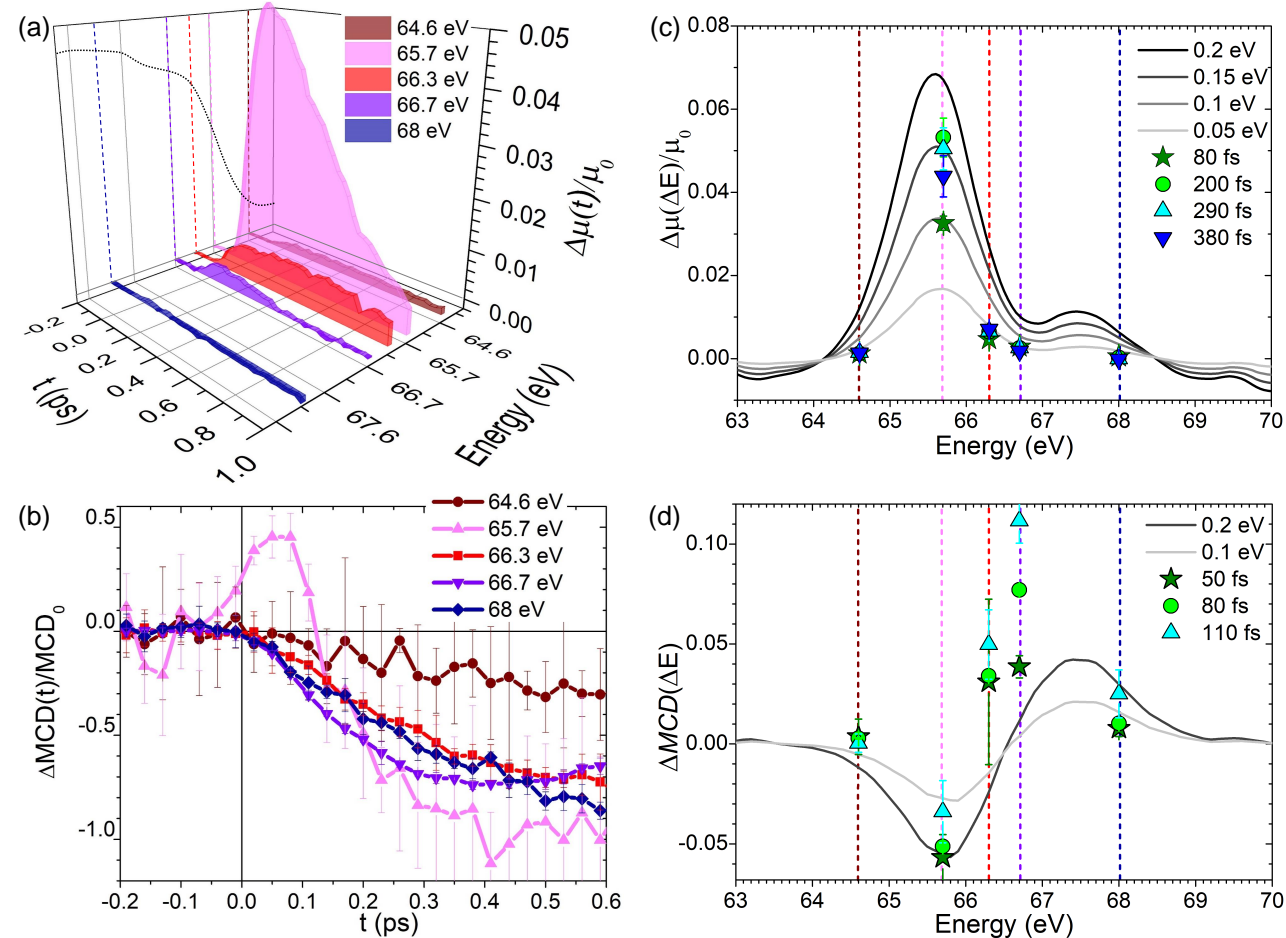

Figure 4. Time- and energy-dependent absorption and MCD data: (a) Relative absorption time traces as a function of probing photon energies and time delay. (b) Relative MCD time traces for the five probing energies. (c) Calculated absorption, as a function of energy, resulting from a shift of the absorption spectra to lower energies (dark and grey lines). The experimental symbols extracted from (a) for different delays are compatible with a maximum shift of $-0.15 \mathrm{eV}$. (d) Calculated magnetization, as a function of energy, considering a similar rigid MCD spectrum red shift (dark and grey lines). The experimental data, plotted for three different delays, are only in agreement with the prediction resulting from a MCD spectrum shift on very short time scales $(<80 \mathrm{fs})$.

Several conclusions can be drawn from these graphs: (i) The relative absorption dynamics depend strongly on the probing energy. In fact, the maximum of the relative transient absorption change observed at $65.7 \mathrm{eV}$ is one order of magnitude stronger than for all other energies scrutinized in this experiment. (ii) The relative magnetization curves display more subtle differences. The characteristic demagnetization times and rates show a weak dependence on the probing energy. As already highlighted, we see that for 66.3 and $64.6 \mathrm{eV}$, the onset of the demagnetization process is delayed with respect to $t=0$. For a probing energy of $65.7 \mathrm{eV}$, we see a surprising increase and subsequent decrease of the magnetization in less than $100 \mathrm{fs}$. Note that this behavior is seen independently of the helicity of the incoming XUV pulses and has been observed in other Ni samples probed during the same experiment (data not shown). 


\section{Discussion}

When a metallic thin film is excited by an ultrashort optical pulse, the electronic population close to the Fermi level is modified, causing a change in the X-ray absorption spectra on femtosecond timescales. This was first measured at the $\mathrm{Ni} L_{3}$-edge by Stamm et al. [22], who suggested that optical pumping of the sample can induce a redshift of the absorption edge during the first hundreds of femtoseconds. The effect was theoretically underpinned by Carva et al. [24], who explained the observed phenomenon as resulting from an optical excitation-induced electron depletion close to the Fermi energy. Furthermore, their calculations suggested that, in addition to the pure absorption spectra, the MCD should also be affected on sub-picosecond timescales. While subsequent experiments remained limited to the $L$-edges of transition metals [17], very recent work by Yao et al. [34] and Rösner et al. [30] unveiled that similar effects can also be observed at the $M$-edges of $\mathrm{Fe}, \mathrm{Co}$, and Ni. Furthermore, both studies demonstrated that, depending on the probing energy, a delay between the magnetic and pure charge dynamics (up to $100 \mathrm{fs}$ ) can exist. As detailed in Yao's study [34], this might hint at a pump-induced redshift of the MCD spectra.

To test this hypothesis, we calculated the $\frac{\Delta \mu(\Delta E)}{\mu_{0}}$ and $\Delta M C D(\Delta E)$ (using the static data shown in Figure $2 b$ ), for different redshift values $\Delta E$ ranging from $0.05 \mathrm{eV}$ up to $0.2 \mathrm{eV}$ (see lines in Figure 4c,d). On top of these computed curves (black to gray lines), we have plotted our experimental data using color symbols for different time delays. When considering absorption changes as a function of energy, our experimental results are in reasonable agreement with the redshift predictions (Figure 4c). The maximum change in absorption is obtained using $E=65.7 \mathrm{eV}$ after $200 \mathrm{fs}$ and corresponds to $\Delta E_{\max }=-0.15 \mathrm{eV}$ with respect to the static spectrum. Note that this is in good agreement with the aforementioned $L_{3}$-edge measurements of Stamm et al., who reported an XAS shift of $-0.13 \mathrm{eV}$ after $200 \mathrm{fs}$ [22], as well as with the calculation presented in the Yao et al. study [34]. All other probed energies give rise to less dramatic changes, in qualitative agreement with our calculations. However, from a quantitative perspective, the observed magnitudes of $\frac{\Delta \mu(\Delta E)}{\mu_{0}}$ show slight deviations from the simple rigid edge shift, which hints at more subtle transient modifications of the absorption spectrum [34].

A comparison of the calculated $\triangle M C D(\triangle E)$ with our experimental data, Figure $4 \mathrm{~d}$, also provides support for a shift of the MCD. Note that since the MCD is inferior to 0 , a negative value of $\triangle M C D(\triangle E)$ corresponds to an increase in magnetization. Therefore, we can interpret the observed increase in the magnetization shortly after $t=0(E=65.7 \mathrm{eV}$, magenta data in panel (b)), as well as the small delays between $t=0$ and the onset of the demagnetization process $(E=64.6 \mathrm{eV}$ and, to a lesser extent, $E=66.3 \mathrm{eV})$ as resulting from a redshift of the MCD. This increase in the magnetization competes with the usual ultrafast demagnetization, and is visible only briefly after $t=0$. Note that this explanation could provide additional insight into a recent study where an increase of the $\mathrm{Ni}$ magnetization on early timescales, in an FeNi alloy, has been attributed to optically induced spin transfer effects [44]. Furthermore, it is in agreement with the theoretical calculation of Carva et al. [24]. For $t>100 \mathrm{fs}$ the magnetization quenching dominates, irrespective of the energy. For probing energies where the aforementioned electronic redistribution and ultrafast demagnetization channels do not compete $(66.7 \mathrm{end} 68 \mathrm{eV})$, we do not find any lag between the onset of the electronic and magnetic dynamics (Figure 3a,b). The increase and delay in the magnetic response briefly after $t=0$ thus unravel a subtle interplay between electronic and spin dynamics. Indeed, this highlights that the electronic population dynamics are spin-dependent and that ultrafast demagnetization is intimately linked to the change in density of states close to the Fermi level as previously observed in photoelectron experiments [26,36].

The redshift of the MCD should also be explicitly observed when plotting the MCD as a function of the photon energy for different delays. Even though we were not able to measure the full spectra, but only five energies, as shown in Figure 5, we indeed find evidence for a slight shift of the static MCD spectrum (black curve) towards smaller energies for $t=80 \mathrm{fs}$ (green stars). On longer timescales, however, the MCD magnitude decreases 
and we observe a distortion of the spectra, especially for higher energies. We emphasize that these results are not at odds with data presented recently by Stamm et al. [31]. In fact, their measurements on $\mathrm{Ni}$ are performed 0.7 ps after the excitation, which is well beyond the typical time where MCD energy shifts can be observed, as shown in the present study. However, this recent work of Stamm et al. [31] demonstrates that it is now possible to measure full MCD spectra for different delays.

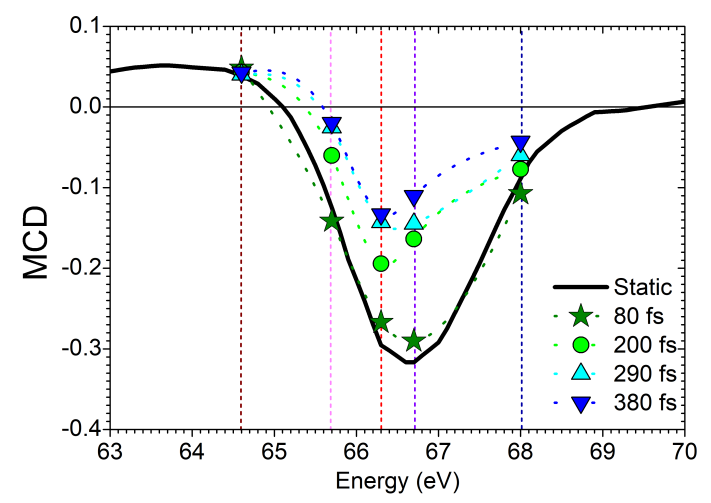

Figure 5. Calculated static MCD spectra (black line) and measured MCD spectra for different time delays (colored symbols).

On longer timescales, as already mentioned, clear differences between the different demagnetization curves become apparent (Figure 4b). Despite the large experimental errors (linked to the use of different CCDs for the measurement of the incoming XUV intensity), which impede a thorough quantitative analysis, it is obvious that the demagnetization observed for $E=64.6 \mathrm{eV}$ is significantly slower than for all other probing energies. This is in agreement with work of Gort et al. [36], who conclude that the characteristic demagnetization times depend on the binding energy of the probed electrons with respect to the Fermi level. As already suggested by Carva [24], "correctly" probing the magnetization might thus require an integration over the entire MCD spectrum (as intrinsically performed using broadband sources), and must be critically discussed in femtomagnetism experiments, where single, well-defined probing wavelengths are used. This could explain why several studies, despite using comparable experimental conditions, reported rather different characteristic demagnetization times [45-47] and significant differences concerning the onset of demagnetization in material systems composed of multiple elements [48-50].

\section{Conclusions}

Using a recently developed X-ray streaking technique $[29,30]$, we have probed the photo-absorption cross-section of a $\mathrm{Ni}$ thin film at the $\mathrm{Ni} \mathrm{M}_{2,3}$-edges using circularly polarized XUV pulses for five probing energies, and both helicities and directions of the applied magnetic field. This allowed us to measure the time-dependent absorption and magnetic circular dichroism (MCD) time traces, and to retrieve information about transient changes occurring in the electronic and spin sub-systems close to the Fermi level. Our results show that the absorption and MCD dynamics both depend on the probing XUV wavelength. While providing a detailed microscopic model that can account for our observations is well beyond the scope of the present paper, we see that, to a first approximation, a redshift of the absorption and MCD spectra on very short timescales ( $<100-200 \mathrm{fs}$ ) can rationalize our data, which is in agreement with recent findings [34]. However, our analysis also hints at more complex modifications of those spectra, and we hope that our experimental findings will stimulate further work in order to obtain a truly quantitative picture of femtosecond charge and spin dynamics in magnetic thin films excited by ultrashort laser pulses. 
Author Contributions: B.R., J.L., C.D., F.C., R.J., B.V., and E.J. conceived the experiments, B.R., F.D. V.A.G., M.L., B.W., and C.D. fabricated the zone plate optics, B.R., R.D., M.L., J.L., A.M., D.N., I.P.N., I.L.-Q., E.P., T.S., M.Z., C.D., F.C., B.V., and E.J. performed the experiment at FERMI. M.H. (Michel Hehn) and G.M. grew the sample. M.H. (Marcel Hennes), V.C., A.K., J.L., F.C., G.S.C., B.V., and E.J. interpreted and discussed the results. M.H. (Marcel Hennes) and E.J. prepared the figures and wrote the manuscript. All authors have read and agreed to the published version of the manuscript.

Funding: This work was funded within the EU-H2020 Research and Innovation Programme, No. 654360 NFFA-Europe (BR). The authors are grateful for the financial support received from CNRSMOMENTUM, the SNSF project (No. 200021-160186), UMAMI ANR-15-CE24-0009, and the CNRSPICS programs.

Institutional Review Board Statement: Not applicable.

Informed Consent Statement: Not applicable.

Data Availability Statement: The data presented in this study are available on request from the corresponding author.

Acknowledgments: We acknowledge the great support of the technical team of FERMI, at Trieste, as well as the help of Hugues RINGUENET and Pascal LEROY from Sorbonne Université. We also thank Clemens von Korff Schmising for stimulating discussions.

Conflicts of Interest: The authors declare no conflict of interest. The funders had no role in the design of the study; in the collection, analysis, or interpretation of data; in the writing of the manuscript, or in the decision to publish the results.

$\begin{array}{ll}\text { Abbreviations } \\ \text { The following abbreviations are used } \\ \text { XUV } & \text { Extreme ultraviolet } \\ \text { MCD } & \text { Magnetic circular dichroism } \\ \text { FEL } & \text { Free electron laser } \\ \text { HHG } & \text { High harmonic generation } \\ \text { CCD } & \text { Charge-coupled device } \\ \text { I } & \text { Intensity } \\ \mathrm{H} & \text { Applied magnetic field } \\ \mathrm{T} & \text { Transmission } \\ \mu & \text { Absorption }\end{array}$

\section{References}

1. Beaurepaire, E.; Merle, J.C.; Daunois, A.; Bigot, J.Y. Ultrafast spin dynamics in ferromagnetic nickel. Phys. Rev. Lett. 1996, 76, 4250. [CrossRef] [PubMed]

2. Kirilyuk, A.; Kimel, A.V.; Rasing, T. Ultrafast optical manipulation of magnetic order. Rev. Mod. Phys. 2010, 82, 2731. [CrossRef]

3. Koopmans, B.; Malinowski, G.; Dalla Longa, F.; Steiauf, D.; Fähnle, M.; Roth, T.; Cinchetti, M.; Aeschlimann, M. Explaining the paradoxical diversity of ultrafast laser-induced demagnetization. Nat. Mater. 2010, 9, 259-265. [CrossRef] [PubMed]

4. Battiato, M.; Carva, K.; Oppeneer, P.M. Superdiffusive spin transport as a mechanism of ultrafast demagnetization. Phys. Rev. Lett. 2010, 105, 027203. [CrossRef] [PubMed]

5. Bierbrauer, U.; Weber, S.T.; Schummer, D.; Barkowski, M.; Mahro, A.K.; Mathias, S.; Schneider, H.C.; Stadtmüller, B.; Aeschlimann, M.; Rethfeld, B. Ultrafast magnetization dynamics in Nickel: impact of pump photon energy. J. Phys. Condens. Matter 2017, 29, 244002. [CrossRef]

6. $\quad$ Ferté, T.; Bergeard, N.; Le Guyader, L.; Hehn, M.; Malinowski, G.; Terrier, E.; Otero, E.; Holldack, K.; Pontius, N.; Boeglin, C. Element-resolved ultrafast demagnetization rates in ferrimagnetic CoDy. Phys. Rev. B 2017, 96, 134303. [CrossRef]

7. Jal, E.; López-Flores, V.; Pontius, N.; Ferté, T.; Bergeard, N.; Boeglin, C.; Vodungbo, B.; Lüning, J.; Jaouen, N. Structural dynamics during laser-induced ultrafast demagnetization. Phys. Rev. B 2017, 95, 184422. [CrossRef]

8. Stanciu, C.D.; Hansteen, F.; Kimel, A.V.; Kirilyuk, A.; Tsukamoto, A.; Itoh, A.; Rasing, T. All-Optical Magnetic Recording with Circularly Polarized Light. Phys. Rev. Lett. 2007, 99, 047601. [CrossRef]

9. Choi, G.M.; Min, B.C.; Lee, K.J.; Cahill, D.G. Spin current generated by thermally driven ultrafast demagnetization. Nat. Commun. 2014, 5. [CrossRef]

10. El-Ghazaly, A.; Gorchon, J.; Wilson, R.B.; Pattabi, A.; Bokor, J. Progress towards ultrafast spintronics applications. J. Magn. Magn. Mater. 2020, 502, 166478. [CrossRef] 
11. Chakravarty, A.; Mentink, J.H.; Davies, C.S.; Yamada, K.T.; Kimel, A.V.; Rasing, T. Supervised learning of an opto-magnetic neural network with ultrashort laser pulses. Appl. Phys. Lett. 2019, 114, 192407. [CrossRef]

12. Becker, H.; Krückel, C.J.; Van Thourhout, D.; Heck, M.J.R. Out-of-Plane Focusing Grating Couplers for Silicon Photonics Integration with Optical MRAM Technology. IEEE J. Sel. Top. Quantum Electron. 2020, 26, 1-8. [CrossRef]

13. Vodungbo, B.; Gautier, J.; Lambert, G.; Sardinha, A.B.; Lozano, M.; Sebban, S.; Ducousso, M.; Boutu, W.; Li, K.; Tudu, B.; et al. Laser-induced ultrafast demagnetization in the presence of a nanoscale magnetic domain network. Nat. Commun. 2012, 3, 999. [CrossRef] [PubMed]

14. Pfau, B.; Schaffert, S.; Müller, L.; Gutt, C.; Al-Shemmary, A.; Büttner, F.; Delaunay, R.; Düsterer, S.; Flewett, S.; Frömter, R.; et al. Ultrafast optical demagnetization manipulates nanoscale spin structure in domain walls. Nat. Commun. 2012, 3, 1-6. [CrossRef]

15. Graves, C.E.; Reid, A.H.; Wang, T.; Wu, B.; de Jong, S.; Vahaplar, K.; Radu, I.; Bernstein, D.P.; Messerschmidt, M.; Müller, L.; et al. Nanoscale spin reversal by non-local angular momentum transfer following ultrafast laser excitation in ferrimagnetic GdFeCo. Nat. Mater. 2013, 12, 293-298. [CrossRef]

16. Weder, D.; von Korff Schmising, C.; Günther, C.M.; Schneider, M.; Engel, D.; Hessing, P.; Strüber, C.; Weigand, M.; Vodungbo, B.; et al. Transient magnetic gratings on the nanometer scale. Struct. Dyn. 2020, 7, 054501. [CrossRef]

17. Boeglin, C.; Beaurepaire, E.; Halté, V.; López-Flores, V.; Stamm, C.; Pontius, N.; Dürr, H.A.; Bigot, J.Y. Distinguishing the ultrafast dynamics of spin and orbital moments in solids. Nature 2010, 465, 458-461. [CrossRef]

18. Radu, I.; Vahaplar, K.; Stamm, C.; Kachel, T.; Pontius, N.; Dürr, H.A.; Ostler, T.A.; Barker, J.; Evans, R.F.L.; Chantrell, R.W.; et al. Transient ferromagnetic-like state mediating ultrafast reversal of antiferromagnetically coupled spins. Nature 2011, 472, 205-208. [CrossRef]

19. Henighan, T.; Trigo, M.; Bonetti, S.; Granitzka, P.; Higley, D.; Chen, Z.; Jiang, M.; Kukreja, R.; Gray, A.; Reid, A.; et al. Generation mechanism of terahertz coherent acoustic phonons in Fe. Phys. Rev. B 2016, 93, 220301. [CrossRef]

20. Reid, A.H.; Shen, X.; Maldonado, P.; Chase, T.; Jal, E.; Granitzka, P.W.; Carva, K.; Li, R.K.; Li, J.; Wu, L.; et al. Beyond a phenomenological description of magnetostriction. Nat. Commun. 2018, 9, 388. [CrossRef]

21. Dornes, C.; Acremann, Y.; Savoini, M.; Kubli, M.; Neugebauer, M.J.; Abreu, E.; Huber, L.; Lantz, G.; Vaz, C.a.F.; Lemke, H.; et al. The ultrafast Einstein-de Haas effect. Nature 2019, 565, 209. [CrossRef] [PubMed]

22. Stamm, C.; Kachel, T.; Pontius, N.; Mitzner, R.; Quast, T.; Holldack, K.; Khan, S.; Lupulescu, C.; Aziz, E.F.; Wietstruk, M.; et al. Femtosecond modification of electron localization and transfer of angular momentum in nickel. Nat. Mater. 2007, 6, 740-743. [CrossRef] [PubMed]

23. Kachel, T.; Pontius, N.; Stamm, C.; Wietstruk, M.; Aziz, E.F.; Dürr, H.A.; Eberhardt, W.; de Groot, F.M.F. Transient electronic and magnetic structures of nickel heated by ultrafast laser pulses. Phys. Rev. B 2009, 80, 092404. [CrossRef]

24. Carva, K.; Legut, D.; Oppeneer, P.M. Influence of laser-excited electron distributions on the X-ray magnetic circular dichroism spectra: Implications for femtosecond demagnetization in Ni. EPL (Europhys. Lett.) 2009, 86, 57002. [CrossRef]

25. Higley, D.J.; Hirsch, K.; Dakovski, G.L.; Jal, E.; Yuan, E.; Liu, T.; Lutman, A.A.; MacArthur, J.P.; Arenholz, E.; Chen, Z.; et al. Femtosecond X-ray magnetic circular dichroism absorption spectroscopy at an X-ray free electron laser. Rev. Sci. Instrum. 2016, 87, 033110. [CrossRef]

26. Eich, S.; Plötzing, M.; Rollinger, M.; Emmerich, S.; Adam, R.; Chen, C.; Kapteyn, H.C.; Murnane, M.M.; Plucinski, L.; Steil, D.; et al. Band structure evolution during the ultrafast ferromagnetic-paramagnetic phase transition in cobalt. Sci. Adv. 2017, 3, e1602094, [CrossRef]

27. You, W.; Tengdin, P.; Chen, C.; Shi, X.; Zusin, D.; Zhang, Y.; Gentry, C.; Blonsky, A.; Keller, M.; Oppeneer, P.M.; et al. Revealing the Nature of the Ultrafast Magnetic Phase Transition in Ni by Correlating Extreme Ultraviolet Magneto-Optic and Photoemission Spectroscopies. Phys. Rev. Lett. 2018, 121, 077204. [CrossRef]

28. Buzzi, M.; Makita, M.; Howald, L.; Kleibert, A.; Vodungbo, B.; Maldonado, P.; Raabe, J.; Jaouen, N.; Redlin, H.; Tiedtke, K.; et al. Single-shot Monitoring of Ultrafast Processes via X-ray Streaking at a Free Electron Laser. Sci. Rep. 2017, 7, 7253. [CrossRef]

29. Jal, E.; Makita, M.; Rösner, B.; David, C.; Nolting, F.; Raabe, J.; Savchenko, T.; Kleibert, A.; Capotondi, F.; Pedersoli, E.; et al. Single-shot time-resolved magnetic X-ray absorption at a free-electron laser. Phys. Rev. B 2019, 99, 144305, [CrossRef]

30. Rösner, B.; Vodungbo, B.; Chardonnet, V.; Döring, F.; Guzenko, V.A.; Hennes, M.; Kleibert, A.; Lebugle, M.; Lüning, J.; Mahne, N.; et al. Simultaneous two-color snapshot view on ultrafast charge and spin dynamics in a Fe-Cu-Ni tri-layer. Struct. Dyn. 2020, 7, 054302. [CrossRef]

31. Stamm, C.; Murer, C.; Wörnle, M.S.; Acremann, Y.; Gort, R.; Däster, S.; Reid, A.H.; Higley, D.J.; Wandel, S.F.; Schlotter, W.F.; et al. X-ray detection of ultrashort spin current pulses in synthetic antiferromagnets. J. Appl. Phys. 2020, 127, 223902. [CrossRef]

32. Willems, F.; Smeenk, C.T.L.; Zhavoronkov, N.; Kornilov, O.; Radu, I.; Schmidbauer, M.; Hanke, M.; von Korff Schmising, C.; Vrakking, M.J.J.; Eisebitt, S. Probing ultrafast spin dynamics with high-harmonic magnetic circular dichroism spectroscopy. Phys. Rev. B 2015, 92, 220405. [CrossRef]

33. Dewhurst, J.K.; Willems, F.; Elliott, P.; Li, Q.Z.; von Korff Schmising, C.; Strüber, C.; Engel, D.W.; Eisebitt, S.; Sharma, S. Element Specificity of Transient Extreme Ultraviolet Magnetic Dichroism. Phys. Rev. Lett. 2020, 124, 077203. [CrossRef] [PubMed]

34. Yao, K.; Willems, F.; von Korff Schmising, C.; Radu, I.; Strüber, C.; Schick, D.; Engel, D.; Tsukamoto, A.; Dewhurst, J.K.; Sharma, S.; et al. Distinct spectral response in M-edge magnetic circular dichroism. Phys. Rev. B 2020, 102, 100405. [CrossRef] 
35. Frietsch, B.; Bowlan, J.; Carley, R.; Teichmann, M.; Wienholdt, S.; Hinzke, D.; Nowak, U.; Carva, K.; Oppeneer, P.M.; Weinelt, M. Disparate ultrafast dynamics of itinerant and localized magnetic moments in gadolinium metal. Nat. Commun. 2015, 6, 8262. [CrossRef]

36. Gort, R.; Bühlmann, K.; Däster, S.; Salvatella, G.; Hartmann, N.; Zemp, Y.; Holenstein, S.; Stieger, C.; Fognini, A.; Michlmayr, T.U.; et al. Early Stages of Ultrafast Spin Dynamics in a 3d Ferromagnet. Phys. Rev. Lett. 2018, 121, 087206. [CrossRef]

37. Jana, S.; Malik, R.S.; Kvashnin, Y.O.; Locht, I.L.M.; Knut, R.; Stefanuik, R.; Di Marco, I.; Yaresko, A.N.; Ahlberg, M.; Åkerman, J.; et al. Analysis of the linear relationship between asymmetry and magnetic moment at the $M$ edge of $3 d$ transition metals. Phys. Rev. Res. 2020, 2, 013180. [CrossRef]

38. Capotondi, F.; Pedersoli, E.; Mahne, N.; Menk, R.H.; Passos, G.; Raimondi, L.; Svetina, C.; Sandrin, G.; Zangrando, M.; Kiskinova, M.; et al. Invited Article: Coherent imaging using seeded free-electron laser pulses with variable polarization: First results and research opportunities. Rev. Sci. Instrum. 2013, 84, 051301. [CrossRef]

39. Allaria, E.; Appio, R.; Badano, L.; Barletta, W.A.; Bassanese, S.; Biedron, S.G.; Borga, A.; Busetto, E.; Castronovo, D.; Cinquegrana, P.; et al. Highly coherent and stable pulses from the FERMI seeded free-electron laser in the extreme ultraviolet. Nat. Photonics 2012, 6, 699-704. [CrossRef]

40. Wang, T.; Zhu, D.; Wu, B.; Graves, C.; Schaffert, S.; Rander, T.; Müller, L.; Vodungbo, B.; Baumier, C.; Bernstein, D.P.; et al. Femtosecond Single-Shot Imaging of Nanoscale Ferromagnetic Order in Co/Pd Multilayers Using Resonant X-ray Holography. Phys. Rev. Lett. 2012, 108, 267403. [CrossRef]

41. Stöhr, J.; Siegmann, H.C. Magnetism: From Fundamentals to Nanoscale Dynamics; Springer Series in Solid-State Sciences; Springer: Berlin/Heidelberg, Germany, 2006.

42. CXRO. X-ray Interactions with Matter; CXRO: Berkeley, CA, USA, 2008. Available online: https://henke.lbl.gov/optical_constants/ filter2.html (accessed on 30 December 2020).

43. Willems, F.; Sharma, S.; Schmising, C.V.K.; Dewhurst, J.K.; Salemi, L.; Schick, D.; Hessing, P.; Strüber, C.; Engel, W.D.; Eisebitt, S. Magneto-Optical Functions at the $3 p$ Resonances of $\mathrm{Fe}, \mathrm{Co}$, and Ni: Ab initio Description and Experiment. Phys. Rev. Lett. 2019, 122, 217202. [CrossRef] [PubMed]

44. Hofherr, M.; Häuser, S.; Dewhurst, J.K.; Tengdin, P.; Sakshath, S.; Nembach, H.T.; Weber, S.T.; Shaw, J.M.; Silva, T.J.; Kapteyn, H.C.; et al. Ultrafast optically induced spin transfer in ferromagnetic alloys. Sci. Adv. 2020, 6, eaay8717. [CrossRef] [PubMed]

45. López-Flores, V.; Bergeard, N.; Halté, V.; Stamm, C.; Pontius, N.; Hehn, M.; Otero, E.; Beaurepaire, E.; Boeglin, C. Role of critical spin fluctuations in ultrafast demagnetization of transition-metal rare-earth alloys. Phys. Rev. B 2013, 87, 214412. [CrossRef]

46. Fan, G.; Legare, K.; Cardin, V.; Xie, X.; Kaksis, E.; Andriukaitis, G.; Pugzlys, A.; Schmidt, B.E.; Wolf, J.P.; Hehn, M.; et al. Time-resolving magnetic scattering on rare-earth ferrimagnets with a bright soft-X-ray high-harmonic source. arXiv 2019, arXiv:1910.14263.

47. Hennes, M.; Merhe, A.; Liu, X.; Weder, D.; Schmising, C.V.K.; Schneider, M.; Günther, C.M.; Mahieu, B.; Malinowski, G.; Hehn, M.; et al. Laser-induced ultrafast demagnetization and perpendicular magnetic anisotropy reduction in a $\mathrm{Co}_{88} \mathrm{~Tb}_{12}$ thin film with stripe domains. Phys. Rev. B 2020, 102, 174437. [CrossRef]

48. Mathias, S.; La-O-Vorakiat, C.; Grychtol, P.; Granitzka, P.; Turgut, E.; Shaw, J.M.; Adam, R.; Nembach, H.T.; Siemens, M.E.; Eich, S.; et al. Probing the timescale of the exchange interaction in a ferromagnetic alloy. Proc. Natl. Acad. Sci. USA 2012, 109, $4792-4797$. [CrossRef]

49. Radu, I.; Stamm, C.; Eschenlohr, A.; Radu, F.; Abrudan, R.; Vahaplar, K.; Kachel, T.; Pontius, N.; Mitzner, R.; Holldack, K.; et al. Ultrafast and Distinct Spin Dynamics in Magnetic Alloys. SPIN 2015, 5, 1550004. [CrossRef]

50. Eschenlohr, A.; Persichetti, L.; Kachel, T.; Gabureac, M.; Gambardella, P.; Stamm, C. Spin currents during ultrafast demagnetization of ferromagnetic bilayers. J. Phys. Condens. Matter 2017, 29, 384002. [CrossRef] 